\title{
Development of an accessible gene expression bioinformatics pipeline to study driver mutations of colorectal cancer
}

\author{
Lisa van den Driest, ${ }^{1}$ Caroline H Johnson, ${ }^{2}$ Nicholas JW Rattray ${ }^{1}$ and Zahra Rattray ${ }^{1}$
}

1. Strathclyde Institute of Pharmacy and Biomedical Sciences, University of Strathclyde, UK.

2. Yale School of Public Health, Yale University, USA.

Keywords: Colon cancer; Mutation; Databases; Prognosis; Expression

\begin{abstract}
Colorectal cancer (CRC) is a global cause of cancer-related mortality driven by genetic and environmental factors which influence therapeutic outcomes. The emergence of next-generation sequencing technologies enables the rapid and extensive collection and curation of genetic data for each cancer type into clinical gene expression biobanks.

In this study we used a combination of bioinformatics tools to investigate the expression patterns and prognostic significance of two genes, adenomatous polyposis coli (APC) and B-Raf protooncogene (BRAF), that are commonly dysregulated in colon cancer. Subsequently, we investigated the pathways and biomolecular effectors implicated in $A P C$ and BRAF function.

Our results show mutation types, frequency, anatomical location and differential expression patterns for $A P C$ and $B R A F$ between colorectal tumour and matched healthy tissue. The prognostic values of $A P C$ and $B R A F$ was investigated as a function of expression level in CRC and other cancer types.

In the era of precision medicine and with significant advancements in biobanking and data curation, there is significant scope to use existing clinical datasets for evaluating the role of mutational drivers in carcinogenesis. This offers the potential for studying combinations of less well-known genes and the discovery of novel biomarkers or studying the association between various effector proteins and pathways.
\end{abstract}

\section{Introduction}

Colorectal cancer (CRC), also known as bowel cancer, is a type of cancer developed by uncontrolled cell growth in parts of the large intestine or the appendix and is a major cause of global morbidity and mortality. In 2020, CRC was ranked as the third most common cancer with 1,931,590 cases $(10 \%)$ and the second highest mortality rate with 935,173 cases $(9.4 \%)$ worldwide ${ }^{1}$. It is predicted that the number of new cases will rise to approximately 2.5 million in $2035^{2}$. Surgery and chemotherapy are routine standard of care interventions used to treat CRC. Novel improved treatment methods such as targeted therapies against genetic mutations and the rapid development of screening techniques such as (virtual) colonoscopy, sigmoidoscopy, stool-based tests and liquid biopsies have shown to detect the disease sooner and extend overall survival rates for patients with CRC. Overall, the 5 -year survival rate for CRC is $\sim 64 \% .^{3}$

CRC is a heterogeneous disease driven by both genetic instability and environmental factors ${ }^{5,6}$. Major advancements in DNA sequencing technologies are resulting in a rapid expansion of biobanks and repositories for patient tumour biopsies. This significant wealth of data offers scientists the information to examine common gene mutations, their cellular pathways and their influence on tumour growth and development. A major challenge in the treatment of CRC is identifying the 
multiple variables that impact the emergence of metastases and their prognostic implications. Various pathways mediate the initiation, progression, and metastasis of CRC or those eligible in the activation of signalling cascades, contain ideal sites for targeted therapy. Therefore, identification and deeper understanding of common mutations in patients with CRC and their effects on cellular pathways are crucial to better understand and treat the disease ${ }^{7}$.

Studies related to the prevention, treatment and development of CRC use both animal models and cell cultures to mimic the cancer microenvironment for patients with CRC. A review study conducted in 2015 investigated the use of pre-clinical models in CRC by investigating current literature (477 relevant articles). Overall, each reported pre-clinical model showed limitations arising from a lack of spontaneous CRC development, or the requirement for a carcinogen for tumour induction in rodents. Moreover, there is significant inter-animal variability in the development of intestinal tumours for murine pre-clinical models. ${ }^{8}$ Alternatively, freely accessible clinical datasets contain a wealth of data to study expression patterns and evaluate the prognostic significance in of gene mutations in human CRC tissue, with the option to further investigate clinicopathologic parameters such as sex, anatomical location, stage and age.

Two genes important in CRC are the tumour suppressor gene adenomatous polyposis coli (APC) and the oncogene B-Raf proto-oncogene (BRAF), implicated in CRC development ${ }^{7,9}$. The most common CRC activation pathway, which is responsible for $70-80 \%$ of all CRC diagnoses, is triggered due to inactivation of the tumour suppressor gene $A P C$, which has an important role in the wnt signalling pathway, intercellular adhesion, cytoskeleton stabilisation, cell cycle regulation and apoptosis ${ }^{10-12}$. Therefore, truncating mutations of $A P C$ resulting in its inactivation are thought to promote tumorigenesis and adenoma formation, then subsequent mutations to KRAS and TP53 propagate the development of carcinomas, commonly found in left-sided colon cancers (LCCS). However, KRAS mutations are in higher frequency in right-sided colon cancers (RCC), together with mutations in the BRAF gene ${ }^{13}$. Recent epidemiologic studies have demonstrated that the anatomical location of CRC tumours impacts the overall patient survival, as patients with RCC were correlated to a poorer prognostic outcome $\mathrm{e}^{14-16}$. Exploring the genetic and prognostic rationale of these gene mutations is important to identify new biomarkers for diagnosis, develop novel potential drug targets for treatment and explore new effective treatment combinations. In this study we intend to develop an integrated bioinformatics workflow that will blend orthogonal datasets and interrogate the two main cellular pathways as drivers of colon cancer aggressiveness in patient populations.

Figure 1 Genetic and biomolecular differences in colorectal cancer as a function of anatomical location. RCCS occur in the cecum, ascending colon and hepatic flexure. LCCs occur in the splenic flexure, descending, sigmoid and rectosigmoid colon.

To-date, several studies have applied bioinformatics tools to investigate diagnostic and prognostic data, key pathways and important genes causing the progression of CRC. Some of these studies conduct a comparison between expression profiles of samples obtained from patients with CRC to those in normal healthy control ${ }^{17-21}$. Yang et al. used bioinformatic analysis to explore the overexpression or downregulation of microRNAs in CRC tissues and their role in tumorigenesis, invasion, and migration ${ }^{22}$. In another study online accessible databanks were used to study the chromobox family proteins and their role in $\mathrm{CRC}^{23}$. In this study we report the analysis of driver mutations (primarily APC and BRAF) in CRC using existing clinical datasets from a range of publicly available biobanks. 


\section{Methods}

cBioPortal (https://www.cbioportal.org/) is an open access resource for cancer genomics that was originally developed by the Memorial Sloan Kettering Cancer Center ${ }^{24}$. This source was used to investigate common gene mutations found in patients with CRC. A total of 7 studies $(n=2,575)$ covering colorectal adenocarcinoma, metastatic colorectal cancer, colon adenocarcinoma, and colon cancer were explored, and a list of frequent mutated genes was generated ${ }^{25-31}$. Additionally, using the same seven studies, the APC/BRAF gene pairing was selected. Under the 'cancer type summary' tab, 'cancer type' was selected to generate a bar chart showing the alteration frequency in each cancer type.

PrognoScan (http://dna00.bio.kyutech.ac.jp/PrognoScan-cgi/PrognoScan.cgi) is a database for performing meta-analysis of the prognostic value of mutations occurring in cancer through incorporating gene expression studies from multiple sources such as the Gene Expression Omnibus (GEO) and reports from individual labs ${ }^{32}$. It relates gene expression data to prognostic outcomes, which enables the evaluation of potential biomarkers and their role in cancer prognosis. In this study PrognoScan was used to assess the correlation between APC and BRAF mRNA expression levels and patient overall survival (OS), disease free survival (DFS) and disease specific survival (DSS). Output generated from PrognoScan include $P$-values (Cox), hazard ratios and confidence intervals. APC and $B R A F$ were independently entered in the PrognoScan website and the $P$-values (Cox), hazard ratios and confidence intervals were obtained for colorectal cancer studies. R Studio software in combination with the forestplot package were used to generate a forest plot from the values obtained via PrognoScan.

Oncomine (https://www.oncomine.org/resource/login.html) is a bioinformatic platform with an extensive collection of datasets ${ }^{33}$. Analysis of $A P C / B R A F$ mRNA expression pattern was performed selecting the following parameters: gene- $A P C / B R A F$, differential analysis- cancer vs. normal analysis, cancer type- colorectal cancer, sample type- clinical specimen, data type- mRNA. Order by: Underexpression: Gene Rank (APC), Order by: Overexpression: Gene Rank (BRAF). A two-fold change, $P$ value corresponding to $1 \mathrm{E}^{-4}$ and top $10 \%$ gene rank were selected as threshold for this analysis. All statistical analyses containing mRNA reporters 215310_at (APC), 203525_s_at (APC) and 243829_at $(B R A F)$ were directly exported from Oncomine.

KMplot (http://kmplot.com/analysis/index.php? $\mathrm{p}=$ service) is an online survival evaluation platform to enable the meta-analysis of a number of different datasets using gene expression data ${ }^{34}$. The correlation between overall survival and APC/BRAF mRNA expression can be assessed using mRNA Start KM Plotter for pan-cancer, gene symbol- $A P C / B R A F$, select all, select draw Kaplan-Meier plot.

Statistical analysis Comparison of APC/BRAF mRNA expression levels was directly obtained from Oncomine using a Student's paired t-test. The prognostic data obtained from Prognoscan was selecting according to the calculated Cox $P$-values and corresponding Hazard ratios $(95 \%$ confidence interval) for various endpoints (OS, DSS, DFS) that were visualized with a forest plot. For comparison of patient survival endpoints, patients were categorized according to survival and survival difference between high and low expression groups was determined by a log-rank test in PrognoScan. To analyse the prognostic value of $A P C / B R A F$ in the Kaplan-Meier plots, P-values from log-rank analyses were used to compare prognostic endpoints between patient cohorts using the KMplot webpage. False discovery rates below $5 \%$ and $\mathrm{P}<0.05$ were considered as statistically significant for all comparisons. 


\section{Results}

\section{Analysis of APC and BRAF gene mutations in CRC using CBioportal}

We first examined the gene mutations and their frequency of occurrence as a function of colorectal cancer clinicopathologic parameters using seven clinical colorectal cancer studies (2,575 samples) in cBioportal $^{25-31}$.

Figure 2 The distribution of $A P C$ and $B R A F$ mutations occurring in their protein sequence across seven colorectal cancer studies. (A), Lollipop diagram correspond ing colorectal cancer studies selected in cBioportal and the frequency and types of changes occurring. Circles indicate sites in which the mutations occur, and the length of each lollipop represents the number of patients with the specific mutation (B), mRNA expression levels and frequency of mutations (C) for $A P C$ and $B R A F(D)$ and fraction of genome alterations as a function of anatomical site. CRA, Colorectal Adenocarcinoma; CRC, Colorectal Cancer; MCRC, Metastatic Colorectal Cancer; CA, Colon Adenocarcinoma; CC, Colon Cancer; CRAT, Colorectal Adenocarcinoma Triplets.

Across the seven studies surveyed in cBioportal, the percentage of samples in which somatic mutations in $A P C$ occurred was $69.6 \%$, with a corresponding frequency of $13.7 \%$ for samples with $B R A F$ mutations. In the case of $A P C$ mutations this corresponded to 2,452 driver mutations $(2,483$ of these were truncating mutations, 213 missense, 45 splice and 12 fusion) and for $B R A F, 327$ driver mutations ( 355 missense, 3 inframe, and 6 fusion). Most of the mutations located in the APC and $B R A F$ protein sequences (Figure $2 A$ ) were annotated as oncogenic in cBioportal. The bar chart (Figure 2B) obtained from $C B i o P o r t a l$ shows that the mutation frequency of $A P C$ is similar across the seven CRC studies selected, while in the case of BRAF a variation in frequency was observed across the studies examined. Significant variation was observed in the frequency (Figure $\mathbf{2 C}$ ) and proportion of mutation types observed across the studies as a function of the tumour site (Figure 2D).

\section{$A P C$ and BRAF mRNA expression in healthy colon tissue and distinct types of CRC tissue representing three reporters (203525_S_at, 215310_at and 243829_at).}

Using Oncomine, three studies (Skrzypczak et al., Kaiser et al. and Hong et al.) were analysed to compare the relative expression patterns of $A P C$ and BRAF mRNA expression levels in healthy colon and tumour tissue based on their anatomical location ${ }^{35-37}$.

Figure 3 APC and BRAF mRNA expression levels in normal colon and colorectal cancer tissue ${ }^{32}$. Box plots ( $A, C, E, G$ and I) on the left side indicate 203525_s_at APC reporter. Box plots (B, D, F, $H$ and J) on the right site represent 215310_at APC reporter. Box plots ( $K$ and $L$ ) down represent 243829_at BRAF reporter. All box plots are located from Oncomine searches. Corresponding datasets included: (A-F) Skrzypczak Colorectal $2(n=40)$, ( $G$ and H) Kaiser Colon ( $n=105)$, (I and J) Hong Colorectal ( $n=82$ ), (K and L) Skrzypczak Colorectal 2 ( $n=40)$. ${ }^{*} P<0.05,{ }^{* *} P<0.01,{ }^{* * *} P<0.001,{ }^{* * *} P<0.0001$, NS $>0.05$ as determined by a t-test.

Boxplots of $A P C$ (Figure 3A-J) mRNA expression levels obtained from Oncomine show a significant reduction of $A P C$ expression levels in distinct types of CRC tissue when compared to healthy colon tissue. Analyses of $A P C$ mRNA expression level patterns across three studies illustrates varied results when comparing different CRC tissue subtypes. In the case of BRAF (Figure 3K-L), mRNA expression levels are significantly elevated in CRC tissues when compared to healthy colon tissue. 
Corresponding fold-changes in expression levels and other study informationderived from Oncomine are summarized in Table 1.

Table 1 Combined APC and BRAF expression are frequently mutated in CRC upon comparison with healthy colon tissue. Fold-changes in APC and BRAF $M R N A$ expression levels relative to matched healthy colon tissue as a function of CRC tumour subtypes as located in Oncomine.

\begin{tabular}{|c|c|c|c|c|c|c|}
\hline Gene & $\begin{array}{l}\text { Colorectal Cancer } \\
\text { Subtype }\end{array}$ & Reporter & $\begin{array}{l}\text { Fold- } \\
\text { change }\end{array}$ & $P$-value & $\mathrm{N}$ & Study \\
\hline$A P C$ & Colon Carcinoma & 203525_s_at & -6.093 & $1.13 \times 10^{-11}$ & 40 & 35 \\
\hline$A P C$ & Colon Carcinoma & 215310_at & -1.230 & 0.036 & 40 & 35 \\
\hline$A P C$ & Colon Carcinoma & 203525_s_at & -4.320 & $1.84 \times 10^{-10}$ & 40 & 35 \\
\hline$A P C$ & Colon Carcinoma & 215310_at & -1.090 & 0.115 & 40 & 35 \\
\hline$A P C$ & Colon Adenoma & 203525_s_at & -7.941 & $5.48 \times 10^{-7}$ & 40 & 35 \\
\hline$A P C$ & Colon Adenoma & 215310_at & -1.418 & 0.003 & 40 & 35 \\
\hline$A P C$ & $\begin{array}{l}\text { Rectosigmoid } \\
\text { Adenocarcinoma }\end{array}$ & 203525_s_at & -1.601 & $9.16 \times 10^{-5}$ & 105 & 36 \\
\hline$A P C$ & $\begin{array}{l}\text { Rectosigmoid } \\
\text { Adenocarcinoma }\end{array}$ & 215310_at & -1.233 & 0.028 & 105 & 36 \\
\hline$A P C$ & Colon Carcinoma & 203525_s_at & -2.166 & $1.14 \times 10^{-11}$ & 82 & 37 \\
\hline$A P C$ & Colon Carcinoma & 215310_at & -1.211 & 0.228 & 82 & 37 \\
\hline BRAF & Colon Carcinoma & 243829_at & 2.054 & $1.41 \times 10^{-7}$ & 40 & 35 \\
\hline$B R A F$ & Colon Carcinoma & 243829_at & 2.763 & $1.89 \times 10^{-6}$ & 40 & 35 \\
\hline
\end{tabular}

The negative fold-change values under -2 together with $P<0.05$ shows that $A P C$ gene expression is significantly reduced in CRC tumour tissue. In the case of colon carcinoma epithelia, colon carcinoma and colon adenoma epithelia a high negative fold-change is observed, confirming a reduction in APC mRNA expression levels in comparison with rectosigmoid adenocarcinoma. The P-value of reported fold-changes were found to be statistically significant $(P<0.05$ ), except for two cases (Skrzypczak and Hong colon carcinoma), which both applied to the 215310_at reporter. The positive fold-change (>2) in BRAF expression coupled with the significant $P$-values $(P<0.05)$ confirms that the BRAF gene expression is significantly increased in colon carcinoma relative to healthy tissue.

\section{The prognostic value of $A P C$ and $B R A F$ gene mutation}

Pan-Cancer analysis of the prognostic value of $A P C$ and BRAF was performed in KMPlot (21 cancer studies) to explore the correlation between patient overall survival and the mRNA expression levels of these actionable genes.

Following analysis of pan-cancer patterns of mRNA expression for $A P C$ and $B R A F$, four datasets were analysed to explore their prognostic value in CRC. Meta-analysis of APC mRNA expression levels and their impact on specific endpoints (including disease-specific survival (DSS), disease-free survival (DFS) and overall survival (OS)) was performed and visualised using forest plots. 
Figure 4 Pan-cancer Kaplan-Meier plots of overall survival correlated with APC mRNA expression levels. Breast cancer $(n=1090, F D R>50)(A)$, esophageal adenocarcinoma $(n=80, F D R=50)(B)$, head-neck squamous cell carcinoma $(n=500$, FDR $>50)(C)$, renal cell carcinoma $(n=530, F D R=1)(D)$, lung squamous cell carcinoma $(n=501, F D R>50)(E)$, and ovarian cancer $(n=374, F D R>50)(F)$, and thyroid cancer ( $n=502$, FDR $>50 \%$ ) (G). Forest plot showing the prognostic value of APC across multiple datasets in PrognoScan. Prognostic endpoints investigated are classified as disease-specific survival (DSS), diseasefree survival (DFS) and overall survival (OS) for APC 215310_at reporter ( $H$ ). Grey squares represent the point estimate of the hazard ratio (HR). FDR: False Discovery Rate and HR: Hazard Ratio.

Pan-cancer analysis of the prognostic value of $A P C$ in KMplot, showed no direct association between $A P C$ levels and patient overall survival. In the case of breast (Figure 4A, HR 1.42), oesophageal (Figure 4B, HR 2.17), lung squamous cell carcinomas (Figure 4E, HR 1.5), and thyroid (Figure 4G, HR 2.91) higher $A P C$ levels were associated with poor overall survival. However, for head and neck squamous cell carcinoma (Figure 4C, HR 0), renal cell carcinoma (Figure 4D, HR 0.72), and ovarian (Figure 4F, HR 0.43) cancer higher APC expression levels were associated with more favourable patient overall survival outcomes.

Next, we examined the prognostic value of APC (APC 215310_at and 203525_s_at reporters) in CRC using PrognoScan. Analysis of PrognoScan yielded four datasets that were subsequently applied to determining the prognostic value of $A P C$ and BRAF in CRC across three survival endpoints (DSS, DFS and OS). Except for the GSE17537 dataset (all endpoints), no statistically significant associations were observed between $A P C$ expression and patient survival endpoints.

Figure 5 Pan-cancer Kaplan-Meier plots for BRAF mRNA expression level impact on patient survival endpoints. Breast cancer $(n=1,090, F D R>50 \%)(A)$, head-neck squamous cell carcinoma $(n=500, F D R>50 \%)(B)$, kidney renal cell carcinoma ( $n$ $530, F D R=3 \%)(C)$, hepatocellular carcinoma $(n=371, F D R>50 \%)(D)$, ovarian cancer $(n=374, F D R>50 \%)(E)$, and endometrial carcinoma ( $n=543, F D R>50 \%$ ) (F). Forest plot showing the prognostic value of BRAF across different datasets. Prognostic value classified as disease-specific survival (DSS), disease-free survival (DFS) and overall survival (OS). Grey squares represent the point estimate of the hazard ratio (HR). Solid lines represent $95 \%$ confidence intervals (CI). The grey line oriented at 1 represents null or no difference. Data represents the 243829_at reporter (G). FDR: False discovery rate

Pan-cancer analysis of BRAF prognostic value in KMplot, showed no consistent association between $B R A F$ mRNA expression levels and patient overall survival across all cancer types studied. In the case of breast (Figure 5A, HR 0.67), head and neck squamous cell carcinoma (Figure 5B, HR 0.72), renal cell carcinomas (Figure 5C, HR 0.56) high BRAF expression levels were associated with better patient overall survival. Conversely, for hepatocellular carcinoma (Figure 5D, HR 1.5), ovarian cancer (Figure 5E, HR 1.33), and endometrial carcinoma (Figure 5F, HR 2.14) higher BRAF expression levels were associated with poor patient overall survival.

Using Prognoscan we identified four CRC datasets that were subsequently applied to determining the prognostic value of BRAF in CRC across three survival endpoints (disease-specific survival- DSS, disease free survival-DFS and overall survival-OS). Except for GSE17536 for the disease-free survival endpoint, no statistically significant associations were observed between BRAF expression and survival endpoints.

\section{Discussion}

In this study we investigated the evidence base for $A P C$ and $B R A F$ expression patterns, frequency of mutations and prognostic role using a combination of open-source bioinformatics tools and clinical datasets. Our rationale for selecting these genes for the present study was informed by the established link between their mutation and CRC tumour aggressiveness ${ }^{38-41}$.

Here, we report an accessible bioinformatics pipeline for performing a meta-analysis using freely available online bioinformatics resources (cBioPortal, Prognoscan, Oncomine, KMPlot) to interrogate existing clinical data on $A P C$ and $B R A F$ as actionable drivers of cancer aggressiveness in patients with 
CRC. The implementation of this approach has several advantages, such as refinement and replacement of pre-clinical models for studying the role and significance of these mutations in cancer progression. Developing a deeper understanding of the frequency of mutations, their clinical significance and impact on patient prognosis provides a strong foundation for the careful design of pre-clinical studies examining the role of these mutations in response to novel experimental therapeutics. Moreover, analysis of large patient clinical datasets increases the translational relevance of findings from these studies to humans through analysing pre-existing clinical data. To our knowledge this study is the first report of the application of bioinformatics approaches to the evaluation of the expression and prognostic value of $A P C$ and $B R A F$ in CRC.

The model genes $A P C$ and $B R A F$ were selected to demonstrate the utility of this presented pipeline. $A P C$ and BRAF mutation assessment have been useful and expedient in prognosis of CRC and their relative expression levels and mutational status have been implicated in treatment response ${ }^{7,9}$. In the present study our analysis of existing clinical datasets shows a significant reduction in APC mRNA expression levels that occurs in distinct anatomical regions of colon tumour tissue when compared to healthy colon tissue (Figure 3). APC is a well-recognized tumour suppressor gene which is highly mutated in CRC. Our findings of frequent mutations and downregulation of $A P C$ expression levels are consistent with previous reports that show the role of $A P C$ loss or gain of function in CRC tumorigenesis $^{38}$. The loss of $A P C$ tumour suppressive functions and gain of function from truncated $A P C$ are widely recognized to contribute to the initiation, progression, and maintenance of $C R C^{7,9,28}$. Our analysis of the expression of $B R A F$, demonstrated a significant increase in BRAF expression in $C R C$ tissues relative to healthy tissue. Also, $B R A F$ mutations function as strong negative prognostic markers, relating to the $\mathrm{V} 600 \mathrm{E}$ (valine to glutamate at codon 600 ) mutation that leads to gain of function. These mutations have previously been observed in mismatch repair deficient tumours and right sided colon tumours in older women ${ }^{42-44}$. Co-occurrence of BRAF and KRAS mutations has frequently been implicated in poor prognosis for CRC patients and a marker of resistance to chemotherapy ${ }^{45}$. Our results indicate that the prognostic value of BRAF mutations is not unique to CRC but has also been observed in kidney renal cell carcinoma. However, previous research implies that patients with renal cell carcinoma lack the presence of $B R A F$ mutations, as opposed to patients with $\mathrm{CRC}^{46,47}$. According to the literature BRAF mutations occur frequently in melanoma, ovarian and thyroid cancer $^{48}$.

Following our analyses of gene mutation signatures and expression levels for $A P C$ and $B R A F$, we examined the prognostic value of these genes (Figure 4-5). Our findings show that loss of APC and gain of $B R A F$ both contribute to poorer survival endpoints in patients with $C R C$. These findings are consistent with previous reports.

A limitation of the present study is a lack of stratification of datasets according to patient metadata, clinicopathologic parameters and anatomical location of CRC tumours. The biology underlying left and right sided $C R C$ has been associated with differences in tumour pathology which vary according to patient parameters (e.g. age, sex, lifestyle factors) ${ }^{49}$. The prognostic value of APC and BRAF expression and their role in CRC tumorigenesis has previously been associated with patient sex and location of the CRC tumour, which has implications for establishing the clinical significance of these actionable mutations according to tumour location. For example, BRAF mutations are typically associated with right-sided colon cancer.

Further investigation of the genetic and phenotypic role of $A P C$ and BRAF genes can contribute to the identification of new biomarkers or the development of novel potential drug targets or 
combinations strategies for overcoming chemoresistance, with the lack of requirement for the use of animal models.

The rapid rise in biobanks and online bioinformatic tools, coupled with the increasing the number of parameters available in these biobanks and software can improve future functional analysis and reduce the need for animal models. The pipeline developed in this study can be directly applied to the evaluation of the expression and prognostic value of other actionable genes in cancer.

\section{Conclusion}

In this study we reported analysis of both APC and BRAF mutations, expression profiles and prognostic roles as actionable genes in CRC. Detailed analysis of genomic alterations in CRC can provide novel insights into disease progression, supporting the development of novel drug treatment combinations that will ultimately improve patient outcomes. Findings from this study demonstrate the importance of accessibility to clinical datasets and a pipeline for investigating the role of genes in the diagnosis and therapy of CRC.

\section{Funding}

The authors acknowledge funding of this research by Tenovus Scotland, RSE Research Reboot, and the FRAME internship for Lisa Van Den Driest.

\section{Conflict of Interest}

The authors declare no conflict of interest.

\section{References}

1. Sung, H. et al. Global Cancer Statistics 2020: GLOBOCAN Estimates of Incidence and Mortality Worldwide for 36 Cancers in 185 Countries. CA: A Cancer Journal for Clinicians 71, (2021).

2. Dekker, E., Tanis, P. J., Vleugels, J. L. A., Kasi, P. M. \& Wallace, M. B. Colorectal cancer. The Lancet 394, (2019).

3. Siegel, R. L., Miller, K. D. \& Jemal, A. Cancer statistics, 2019. CA: A Cancer Journal for Clinicians 69, (2019).

4. Xie, Y.-H., Chen, Y.-X. \& Fang, J.-Y. Comprehensive review of targeted therapy for colorectal cancer. Signal Transduction and Targeted Therapy 5, (2020).

5. Binefa, G. Colorectal cancer: From prevention to personalized medicine. World Journal of Gastroenterology 20, (2014).

6. Rattray, N. J. W. et al. Environmental Influences in the Etiology of Colorectal Cancer: the Premise of Metabolomics. Current Pharmacology Reports 3, (2017).

7. Schell, M. J. et al. A multigene mutation classification of 468 colorectal cancers reveals a prognostic role for APC. Nature Communications 7, (2016).

8. Mittal, V. K., Bhullar, J. S. \& Jayant, K. Animal models of human colorectal cancer: Current status, uses and limitations. World Journal of Gastroenterology 21, 1185411861 (2015). 
9. Fennell, L. J. et al. APC Mutation Marks an Aggressive Subtype of BRAF Mutant Colorectal Cancers. Cancers 12, (2020).

10. Morin, P. J., Vogelstein, B. \& Kinzler, K. W. Apoptosis and APC in colorectal tumorigenesis. Proceedings of the National Academy of Sciences 93, (1996).

11. Morrison, E. E., Wardleworth, B. N., Askham, J. M., Markham, A. F. \& Meredith, D. M. EB1, a protein which interacts with the APC tumour suppressor, is associated with the microtubule cytoskeleton throughout the cell cycle. Oncogene 17, (1998).

12. Kawasaki, Y. Asef, a Link Between the Tumor Suppressor APC and G-Protein Signaling. Science 289, (2000).

13. Cai, Y. et al. Sex Differences in Colon Cancer Metabolism Reveal A Novel Subphenotype. Scientific Reports 10, (2020).

14. Petrelli, F. et al. Prognostic Survival Associated With Left-Sided vs Right-Sided Colon Cancer. JAMA Oncology 3, (2017).

15. Gervaz, P. et al. Right colon cancer: Left behind. European Journal of Surgical Oncology (EJSO) 42, (2016).

16. Loupakis, F. et al. Primary Tumor Location as a Prognostic Factor in Metastatic Colorectal Cancer. JNCI: Journal of the National Cancer Institute 107, (2015).

17. Zhao, B. et al. Identification of Potential Key Genes and Pathways in Early-Onset Colorectal Cancer Through Bioinformatics Analysis. Cancer Control 26, (2019).

18. Liang, B., Li, C. \& Zhao, J. Identification of key pathways and genes in colorectal cancer using bioinformatics analysis. Medical Oncology 33, (2016).

19. Chen, L. et al. Identification of biomarkers associated with diagnosis and prognosis of colorectal cancer patients based on integrated bioinformatics analysis. Gene 692, (2019).

20. Sun, G. et al. Identification of differentially expressed genes and biological characteristics of colorectal cancer by integrated bioinformatics analysis. Journal of Cellular Physiology 234, (2019).

21. Liu, X., Liu, X., Qiao, T. \& Chen, W. Identification of crucial genes and pathways associated with colorectal cancer by bioinformatics analysis. Oncology Letters 19, 1881-1889 (2020).

22. Yang, G., Zhang, Y. \& Yang, J. A Five-microRNA Signature as Prognostic Biomarker in Colorectal Cancer by Bioinformatics Analysis. Frontiers in Oncology 9, (2019).

23. Li, Q., Pan, Y., Cao, Z. \& Zhao, S. Comprehensive Analysis of Prognostic Value and Immune Infiltration of Chromobox Family Members in Colorectal Cancer. Frontiers in Oncology 10, (2020).

24. Gao, J. et al. Integrative Analysis of Complex Cancer Genomics and Clinical Profiles Using the cBioPortal. Science Signaling 6, (2013).

25. Vasaikar, S. et al. Proteogenomic Analysis of Human Colon Cancer Reveals New Therapeutic Opportunities. Cell 177, (2019). 
26. Guda, K. et al. Novel recurrently mutated genes in African American colon cancers. Proceedings of the National Academy of Sciences 112, (2015).

27. Yaeger, R. et al. Clinical Sequencing Defines the Genomic Landscape of Metastatic Colorectal Cancer. Cancer Cell 33, (2018).

28. Mondaca, S. et al. Specific Mutations in APC, but Not Alterations in DNA Damage Response, Associate With Outcomes of Patients With Metastatic Colorectal Cancer. Gastroenterology 159, (2020).

29. Brannon, A. R. et al. Comparative sequencing analysis reveals high genomic concordance between matched primary and metastatic colorectal cancer lesions. Genome Biology 15, (2014).

30. Seshagiri, S. et al. Recurrent R-spondin fusions in colon cancer. Nature 488, (2012).

31. Giannakis, M. et al. Genomic Correlates of Immune-Cell Infiltrates in Colorectal Carcinoma. Cell Reports 15, (2016).

32. Mizuno, H., Kitada, K., Nakai, K. \& Sarai, A. PrognoScan: a new database for metaanalysis of the prognostic value of genes. BMC Medical Genomics 2, (2009).

33. Oncomine. in Encyclopedia of Genetics, Genomics, Proteomics and Informatics (Springer Netherlands, 2008). doi:10.1007/978-1-4020-6754-9_11849.

34. Nagy, Á., Munkácsy, G. \& Győrffy, B. Pancancer survival analysis of cancer hallmark genes. Scientific Reports 11, (2021).

35. Skrzypczak, M. et al. Modeling Oncogenic Signaling in Colon Tumors by Multidirectional Analyses of Microarray Data Directed for Maximization of Analytical Reliability. PLOS ONE 5, (2010).

36. Kaiser, S. et al. Transcriptional recapitulation and subversion of embryonic colon development by mouse colon tumor models and human colon cancer. Genome Biology 8, (2007).

37. Hong, Y., Downey, T., Eu, K. W., Koh, P. K. \& Cheah, P. Y. A 'metastasis-prone' signature for early-stage mismatch-repair proficient sporadic colorectal cancer patients and its implications for possible therapeutics. Clinical \& Experimental Metastasis 27, (2010).

38. Zhang, L. \& Shay, J. W. Multiple Roles of APC and its Therapeutic Implications in Colorectal Cancer. JNCl: Journal of the National Cancer Institute 109, (2017).

39. Thiel, A. \& Ristimäki, A. Toward a Molecular Classification of Colorectal Cancer: The Role of BRAF. Frontiers in Oncology 3, (2013).

40. Aghabozorgi, A. S. et al. Role of adenomatous polyposis coli (APC) gene mutations in the pathogenesis of colorectal cancer; current status and perspectives. Biochimie 157, (2019).

41. Sanz-Garcia, E., Argiles, G., Elez, E. \& Tabernero, J. BRAF mutant colorectal cancer: prognosis, treatment, and new perspectives. Annals of Oncology 28, (2017). 
42. Fariña-Sarasqueta, A. et al. The BRAF V600E mutation is an independent prognostic factor for survival in stage II and stage III colon cancer patients. Annals of Oncology 21, (2010).

43. Thiel, A. \& Ristimäki, A. Toward a Molecular Classification of Colorectal Cancer: The Role of BRAF. Frontiers in Oncology 3, (2013).

44. Samowitz, W. S. et al. Poor Survival Associated with the BRAF V600E Mutation in Microsatellite-Stable Colon Cancers. Cancer Research 65, (2005).

45. Midthun, L. et al. Concomitant KRAS and BRAF mutations in colorectal cancer. Journal of Gastrointestinal Oncology 10, (2019).

46. Gattenlöhner, S., Etschmann, B., Riedmiller, H. \& Müller-Hermelink, H.-K. Lack of KRAS and BRAF Mutation in Renal Cell Carcinoma. European Urology 55, (2009).

47. Bayrak, O. et al. Evaluation of EGFR, KRAS and BRAF gene mutations in renal cell carcinoma. Journal of Kidney Cancer and VHL 1, (2014).

48. Dhomen, N. \& Marais, R. New insight into BRAF mutations in cancer. Current Opinion in Genetics \& Development 17, 31-39 (2007).

49. Bylsma, L. C. et al. Prevalence of RAS and BRAF mutations in metastatic colorectal cancer patients by tumor sidedness: A systematic review and meta-analysis. Cancer Medicine 9, (2020).

\section{List of Figures}

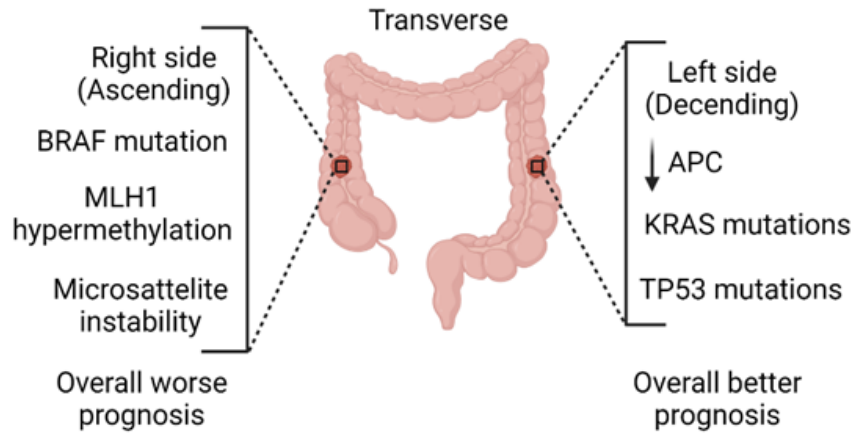

Figure 1 Genetic and biomolecular differences in colorectal cancer as a function of anatomical location. RCCS occur in the cecum, ascending colon and hepatic flexure. LCCs occur in the splenic flexure, descending, sigmoid and rectosigmoid colon. 
medRxiv preprint doi: https://doi.org/10.1101/2021.11.10.21266196; this version posted November 15, 2021. The copyright holder for this preprint (which was not certified by peer review) is the author/funder, who has granted medRxiv a license to display the preprint in perpetuity.

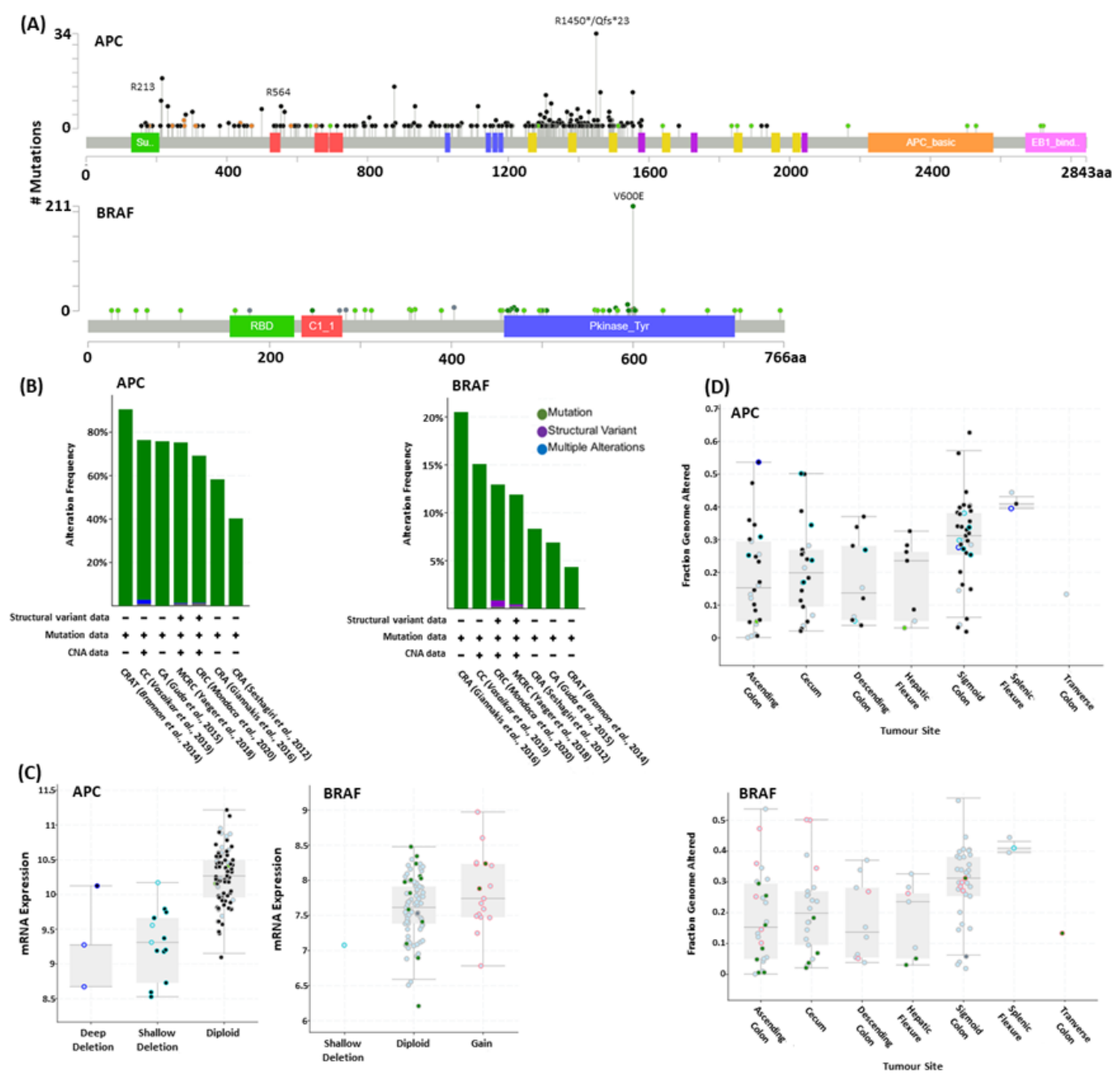

Figure 2 The distribution of $A P C$ and $B R A F$ mutations occurring in their protein sequence across seven colorectal cancer studies. (A), Lollipop diagram corresponding colorectal cancer studies selected in cBioportal and the frequency and types of changes occurring. Circles indicate sites in which the mutations occur, and the length of each lollipop represents the number of patients with the specific mutation (B), mRNA expression levels and frequency of mutations (C) for APC and $B R A F(D)$ and fraction of genome alterations as a function of anatomical site. CRA, Colorectal Adenocarcinoma; CRC, Colorectal Cancer; MCRC, Metastatic Colorectal Cancer; CA, Colon Adenocarcinoma; CC, Colon Cancer; CRAT, Colorectal Adenocarcinoma Triplets. 
medRxiv preprint doi: https://doi.org/10.1101/2021.11.10.21266196; this version posted November 15, 2021. The copyright holder for this preprint (which was not certified by peer review) is the author/funder, who has granted medRxiv a license to display the preprint in perpetuity.

All rights reserved. No reuse allowed without permission.
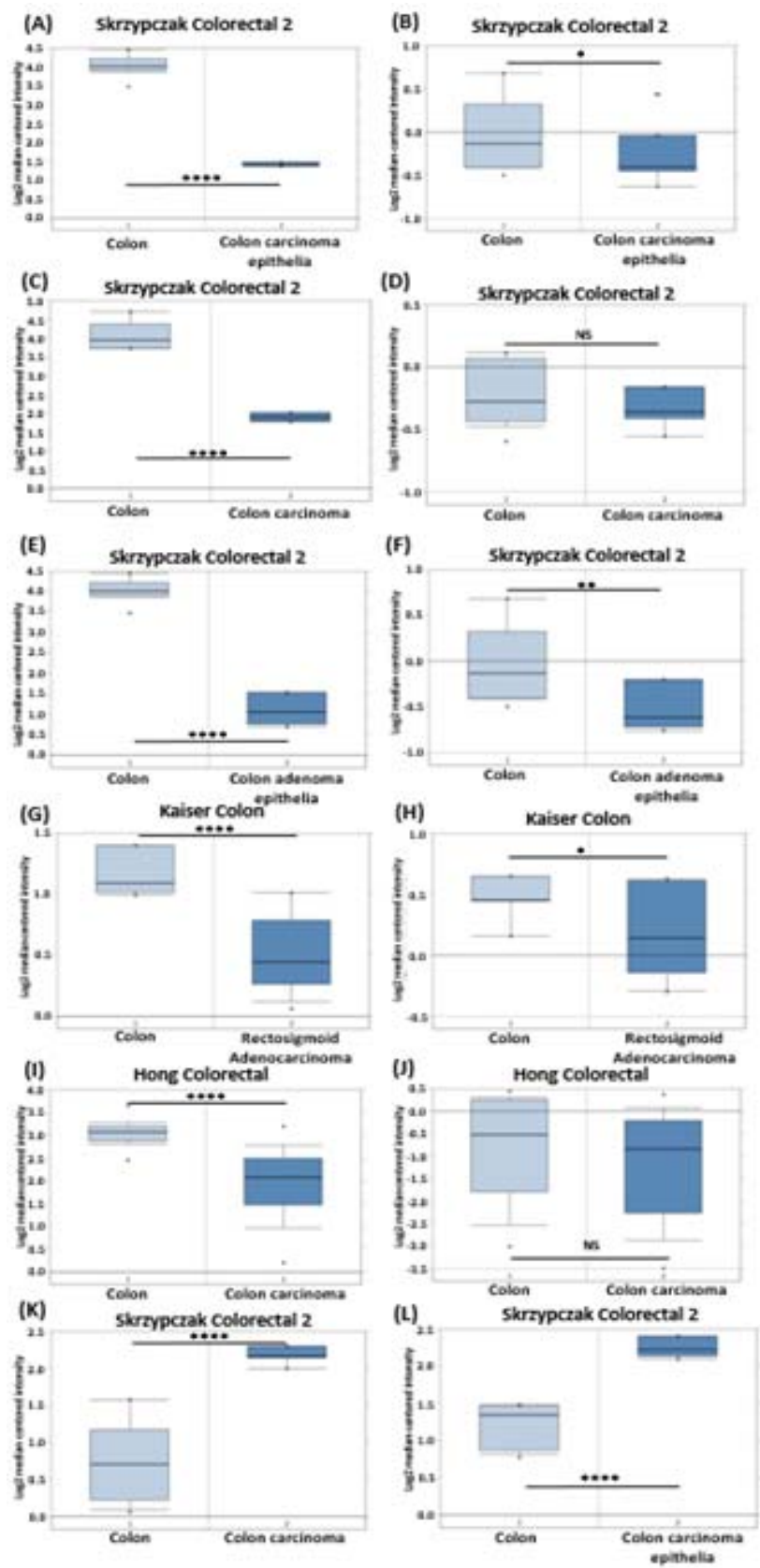

Figure 3 APC and BRAF mRNA expression levels in normal colon and colorectal cancer tissue ${ }^{32}$. Box plots (A, C, E, G and I) on the left side indicate 203525_s_at APC reporter. Box plots (B, D, F, H and J) on the right site represent 215310_at APC reporter. Box plots ( $K$ and $L$ ) down represent 243829_at BRAF reporter. All box plots are located from Oncomine searches. Corresponding datasets included: (A-F) Skrzypczak Colorectal 2 ( $n=40)$, ( $G$ and H) Kaiser Colon ( $n=105)$, (I and J) Hong Colorectal ( $n=82$ ), (K and L) Skrzypczak Colorectal 2 ( $n=40)$. ${ }^{*} P<0.05,{ }^{* *} P<0.01, * * * P<0.001, * * * *<0.0001, N S>0.05$ as determined by a t-test. 
medRxiv preprint doi: https://doi.org/10.1101/2021.11.10.21266196; this version posted November 15, 2021. The copyright holder for this preprint (which was not certified by peer review) is the author/funder, who has granted medRxiv a license to display the preprint in perpetuity.

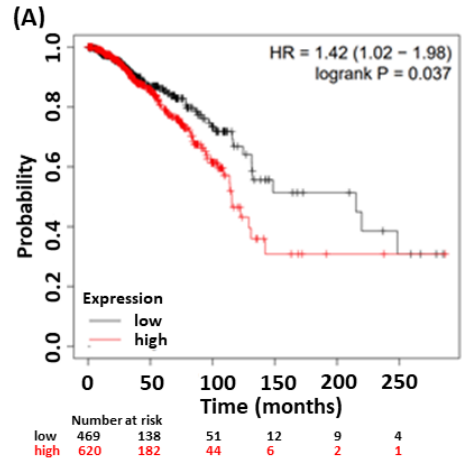

(D)

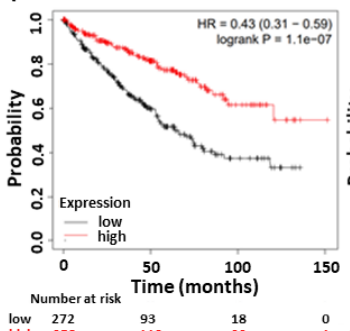

(E)
(B)

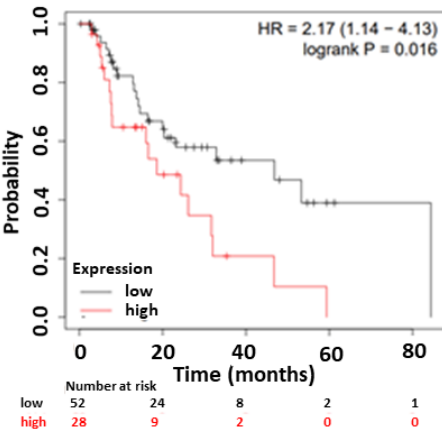

(C)

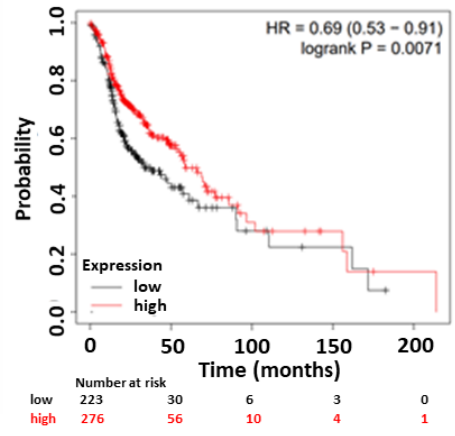

(F)

(G)
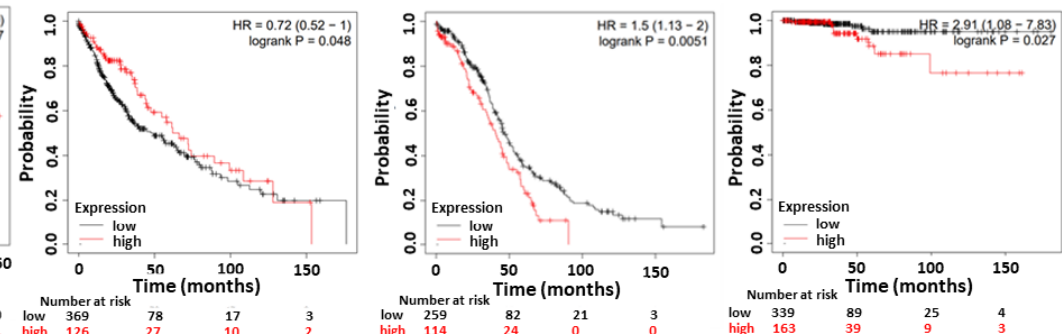

(H)

H) Endpoint Dataset

\begin{tabular}{llll}
\hline DSS & GSE17536 & 177 & 0.390
\end{tabular}

DSS GSE17537

49

0.0124

$-$

DFS GSE12945

$51 \quad 0.187$

DFS

GSE14333

226

0.181

$-$

DFS

GSE17536

145

0.731

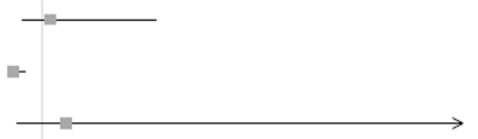

$\mathrm{HR}(95 \% \mathrm{Cl})$

DFS

GSE17537

55

0.00751

GSE12945

0.643

os

GSE17536

177

0.883

GSE17537

55

0.0330

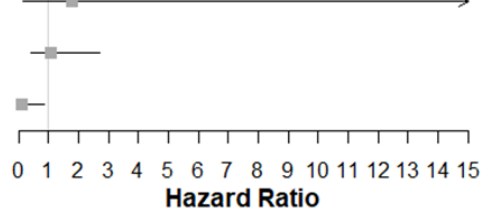

$1.60[0.55-4.65]$

$0.02[0.00-0.44]$

9.91 [0.33 - 299.02]

$1.23[0.91-1.65]$

$1.26[0.33-4.77]$

$0.03[0.00-0.40]$

1.79 [0.15 - 20.87]

$1.07[0.42-2.71]$

$0.10[0.01-0.83]$

Hazard Ratio

Figure 4 Pan-cancer Kaplan-Meier plots of overall survival correlated with APC mRNA expression levels. Breast cancer $(\mathrm{n}=1090, \mathrm{FDR}>50)(\boldsymbol{A})$, esophageal adenocarcinoma $(n=80, F D R=50)(\boldsymbol{B})$, head-neck squamous cell carcinoma $(\mathrm{n}=500, \mathrm{FDR}$ $>50)(C)$, renal cell carcinoma $(n=530, F D R=1)(D)$, lung squamous cell carcinoma $(n=501, F D R>50)(E)$, and ovarian cancer $(n=374, F D R>50)(F)$, and thyroid cancer $(n=502$, FDR $>50 \%)(G)$. Forest plot showing the prognostic value of APC across multiple datasets in PrognoScan. Prognostic endpoints investigated are classified as disease-specific survival (DSS), diseasefree survival (DFS) and overall survival (OS) for APC 215310_at reporter (H). Grey squares represent the point estimate of the hazard ratio (HR). FDR: False Discovery Rate and HR: Hazard Ratio. 
medRxiv preprint doi: https://doi.org/10.1101/2021.11.10.21266196; this version posted November 15, 2021. The copyright holder for this preprint (which was not certified by peer review) is the author/funder, who has granted medRxiv a license to display the preprint in perpetuity.

All rights reserved. No reuse allowed without permission.
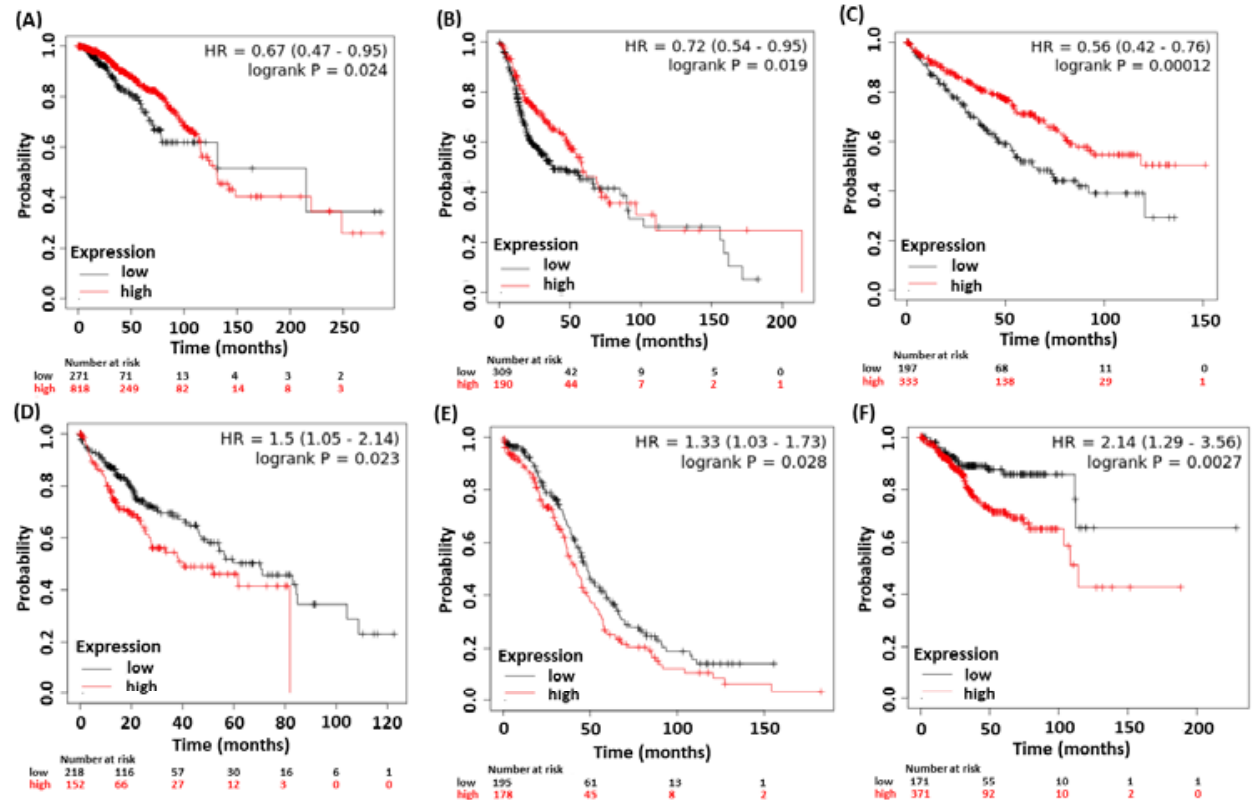

(G)

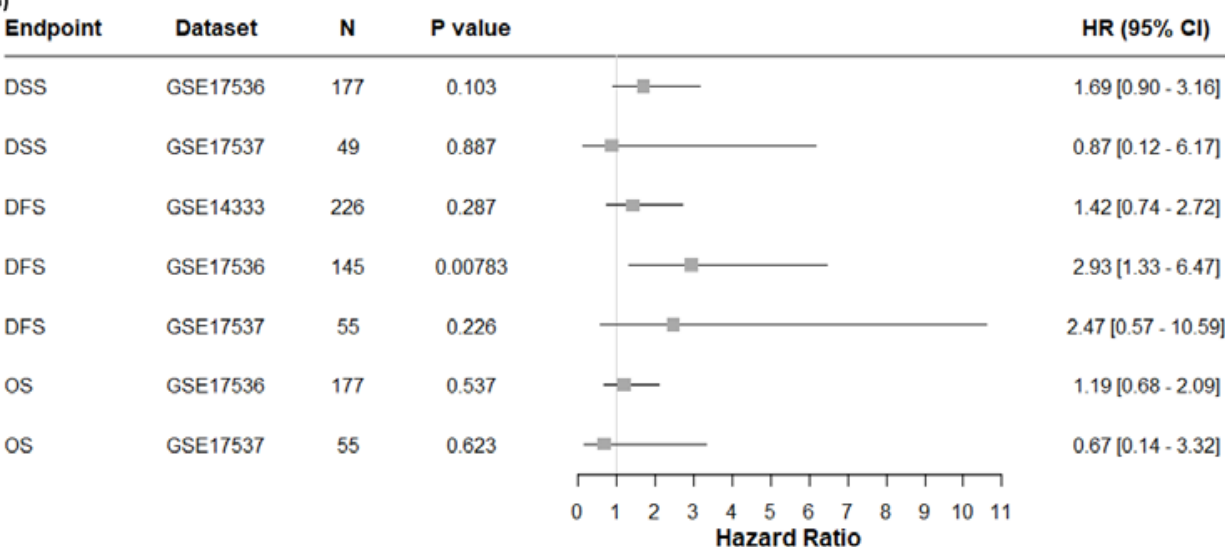

Figure 5 Pan-cancer Kaplan-Meier plots for BRAF mRNA expression level impact on patient survival endpoints. Breast cancer $(n=1,090, F D R>50 \%)(A)$, head-neck squamous cell carcinoma ( $n=500, F D R>50 \%)(B)$, kidney renal cell carcinoma ( $n$ $530, F D R=3 \%)(C)$, hepatocellular carcinoma $(n=371, F D R>50 \%)(D)$, ovarian cancer $(n=374, F D R>50 \%)(E)$, and endometrial carcinoma ( $n=543, F D R>50 \%$ ) (F). Forest plot showing the prognostic value of BRAF across different datasets. Prognostic value classified as disease-specific survival (DSS), disease-free survival (DFS) and overall survival (OS). Grey squares represent the point estimate of the hazard ratio (HR). Solid lines represent $95 \%$ confidence intervals (CI). The grey line oriented at 1 represents null or no difference. Data represents the 243829_at reporter (G). FDR: False discovery rate 
medRxiv preprint doi: https://doi.org/10.1101/2021.11.10.21266196; this version posted November 15, 2021. The copyright holder for this preprint (which was not certified by peer review) is the author/funder, who has granted medRxiv a license to display the preprint in perpetuity.

All rights reserved. No reuse allowed without permission.

\section{List of Tables}

Table 1 Combined APC and BRAF expression are frequently mutated in CRC upon comparison with healthy colon tissue. Fold-changes in APC and BRAF $M R N A$ expression levels relative to matched healthy colon tissue as a function of CRC tumour subtypes as located in Oncomine.

\begin{tabular}{|c|c|c|c|c|c|c|}
\hline Gene & $\begin{array}{l}\text { Colorectal Cancer } \\
\text { Subtype }\end{array}$ & Reporter & $\begin{array}{l}\text { Fold- } \\
\text { change }\end{array}$ & $P$-value & $\mathrm{N}$ & Study \\
\hline$A P C$ & Colon Carcinoma & 203525_s_at & -6.093 & $1.13 \times 10^{-11}$ & 40 & 35 \\
\hline$A P C$ & Colon Carcinoma & 215310_at & -1.230 & 0.036 & 40 & 35 \\
\hline$A P C$ & Colon Carcinoma & 203525_s_at & -4.320 & $1.84 \times 10^{-10}$ & 40 & 35 \\
\hline$A P C$ & Colon Carcinoma & 215310_at & -1.090 & 0.115 & 40 & 35 \\
\hline$A P C$ & Colon Adenoma & 203525_s_at & -7.941 & $5.48 \times 10^{-7}$ & 40 & 35 \\
\hline$A P C$ & Colon Adenoma & 215310_at & -1.418 & 0.003 & 40 & 35 \\
\hline$A P C$ & $\begin{array}{l}\text { Rectosigmoid } \\
\text { Adenocarcinoma }\end{array}$ & 203525_s_at & -1.601 & $9.16 \times 10^{-5}$ & 105 & 36 \\
\hline$A P C$ & $\begin{array}{l}\text { Rectosigmoid } \\
\text { Adenocarcinoma }\end{array}$ & 215310_at & -1.233 & 0.028 & 105 & 36 \\
\hline$A P C$ & Colon Carcinoma & 203525_s_at & -2.166 & $1.14 \times 10^{-11}$ & 82 & 37 \\
\hline$A P C$ & Colon Carcinoma & 215310_at & -1.211 & 0.228 & 82 & 37 \\
\hline$B R A F$ & Colon Carcinoma & 243829_at & 2.054 & $1.41 \times 10^{-7}$ & 40 & 35 \\
\hline$B R A F$ & Colon Carcinoma & 243829_at & 2.763 & $1.89 \times 10^{-6}$ & 40 & 35 \\
\hline
\end{tabular}

